\title{
The effect of visual information on gait parameters with induced ankle muscle fatigue
}

\author{
Hye Rim Suh ${ }^{a}$, Jin-Hee Hwang ${ }^{b}$, Su-Young Lee ${ }^{c}$ \\ ${ }^{a}$ Department of Physiology, Korea University College of Medicine and Neuroscience Research Institute, Seoul, Republic of Korea \\ ${ }^{b}$ Department of Physical Therapy, Myongii Choonhey Hospital, Seoul, Republic of Korea \\ ${ }^{\mathrm{c} D e p a r t m e n t}$ of Physical Therapy, Division of Health Science, Baekseok University, Cheonan, Republic of Korea
}

Objective: The purpose of this study was to investigate the effect of visual block with ankle joint fatigue on gait and dynamic balance ability.

Design: Cross-sectional study.

Methods: Thirty healthy young adults (men=15, women=15) between 22 to 25 years of age voluntarily participated in this study. All subjects performed the gait and dynamic balance test successively in two conditions: the visual block and the open eyes condition. Before the gait and dynamic balance test, muscle fatigue on the ankle joint was induced to all subjects by doing ankle dorsiflexion and plantarflexion alternately, and then gait parameters (step length, stride length, cadence, velocity, single limb support, and double limb support) were assessed by walking on the GAITRite system (CIR Systems Inc., USA). Subjects also performed the functional reach test (FRT) for assessment of dynamic balance. This study examined gait parameters and FRT scores in each visual block and open eyes condition.

Results: The results showed that FRT scores with the visual block condition significantly decreased compared to without visual blocking $(p<0.01)$. Step length, stride length, cadence, and velocity of gait parameters decreased significantly in the visual block condition $(p<0.01)$ while there was no significant difference for single limb support. However, double limb support increased significantly in the visual block system $(p<0.01)$.

Conclusions: Therefore, blocking of visual information with muscle fatigue of the ankle joint can affect gait and balance ability of young adults and increase the risk of falls.

Key Words: Gait, Muscle fatigue, Postrual balance

\section{Introduction}

The visual system is important in maintaining stability and planning the route in walking. It does so by adjusting the walking speed and the foot position [1]. In addition, vision plays an important role in maintaining balance and posture. In visual blocking, posture is adjusted or balanced through sensory information such as vestibular, proprioceptive, and tactile senses [2]. The visual stabilization of postural fluctuations provides a basis for effectively detecting vibrations in a static environment, and visual information is potentially effective in reducing postural fluctuations [3]. Therefore, elderly people with weak eyesight report that the incidence of falls is about three times higher than elderly people with normal eyesight [4].

One of the essential factors in postural control is the ability to generate enough muscle strength to maintain stability during exercise [5]. The calf muscles play a major role in the postural control because the perpendicular line in the center of the mass falls anteriorly to the ankle joint axis in standing [6]. The instability of the ankle joints increases the postural fluctuation when the stabilizing muscles of the ankle joints

Received: 25 August, 2017 Revised: 14 September, 2017 Accepted: 15 September, 2017

Corresponding author: Su-Young Lee

Department of Physical Therapy, Division of Health Science, Baekseok University, 76 Munam-ro, Dongnam-gu, Cheonan 31065 , Republic of Korea Tel: 82-41-550-2546 Fax: 82-41-550-2829 E-mail: rosei118@bu.ac.kr

(c) This is an Open-Access article distributed under the terms of the Creative Commons Attribution Non-Commercial License (http://creativecommons.org/licens es/by-nc/4.0) which permits unrestricted non-commercial use, distribution, and reproduction in any medium, provided the original work is properly cited.

Copyright $\odot 2017$ Korean Academy of Physical Therapy Rehabilitation Science 
are unable to produce sufficient force [5]. Additionally, fatigue in ankle joint muscles affect joint proprioception and increase the threshold of the muscle spindles, which alters postural control ability and joint position [7]. Therefore, fatigue in postural muscles, such as gastrocnemius and soleus, increases difficulty in sustaining balance and results in reduced postural control [8]. The disability to balance can cause a decrease in ability to walk, and increase the risk in falls for elderly people [9].

Gait is the most basic movement of human beings where the body moves from one point to another [10]. The process of gait is the nervous system and the musculoskeletal system used together continuously and repetitively [11]. As a result, gait is highly correlated with balance ability [12]. In addition, walking ability plays an important independent variable for predicting falls [13], and impaired walking ability is an important factor in causing falls [14]. Symptoms of muscle fatigue are similar to the general symptoms of insufficient strength or to many muscle failure diseases, which is directly related to decrease postural control and balance of patients and the elderly [15]. Muscle fatigue with visual block has been reported to produce posture instability [16]. In previous studies the balance ability was decreased as the age increased, leading to increased risk factors for falling, accompanied by changes in posture and concentration [17].

The effects of ankle or lower muscle fatigue and the effects of visual deficiency on balance have been studied extensively. However, it remains unclear whether visual block with ankle fatigue affects postural control and gait. The purpose of this study was to determine whether ankle muscle fatigue and visual deficiency are risk factors for falls by comparing gait parameters and dynamic balance with or without visual information after inducing muscle fatigue in the ankle joint.

\section{Methods}

\section{Participants}

The subjects of this study were 30 young men ( 15 males, 15 females) attending Baekseok University in Cheonan city, Chungcheongnam-do. The exclusion criteria for subjects of the study were those with musculoskeletal or nervous system lesions, ankle or foot weakness or pain, abnormal cognitive function, and balance or visual impairment. This study was approved by the Institutional Review Board of the Baekseok University (IRB No. BUIRB-201610-HR-020). Subjects voluntarily participate for the study and provided their informed consent after being provided with sufficient details of the study. The general characteristics of participants in this study are shown in Table 1 . The mean age of all participants was 22.8 years, mean height was $164.7 \mathrm{~cm}$, and the mean weight was $56.7 \mathrm{~kg}$.

\section{Experimental procedures}

All subjects performed fatigue-inducing tasks to create ankle fatigue. The induction of fatigue in the subject was confirmed with the rating of perceived exertion (RPE), in which the fatigue-inducing tasks were stopped when subjects reported 17 or more on the RPE to prevent the injury of the calf muscle [18]. After the patient rested while sitting on a chair for 1 minute, their eyes were covered by an eye bandage (at first, to prevent the learning effect) and then gait was measured three times. After resting for 5 minutes, the functional reach test (FRT), a dynamic balance test, was repeated 3 times. Thereafter, in a non-blocked state, gait was measured 3 times, and after 5 minutes of rest, the FRT was repeated 3 times.

\section{Ankle fatigue}

In this study, the fatigue induction of the ankle joint muscles was performed according to the modified method of Boyas et al. [19] to prevent injury of the calf muscle. The fatigue induction was commenced in a standing position while leaning against the wall. At first, subjects were instructed to dorsiflex the ankle and then resume back to the starting position, then to lift the heel and then to return back to the starting position. This was considered as one cycle of ankle joint movement [19]. The speed of one cycle was performed at $4 / 4,40$ beat/min using a metronome. The maximum exercise criteria were: when the range of motion was less than $50 \%$ or when the metronome-based speed was too difficult or could no longer be performed. The subjects were encouraged to perform at least greater than 100 cycles of ankle joint exercises (Figure 1).

Table 1. General characteristics of the participants $\quad(\mathrm{N}=30)$

\begin{tabular}{lcrr}
\hline Characteristic & Female $(\mathrm{n}=15)$ & Male $(\mathrm{n}=15)$ & \multicolumn{1}{c}{ Total } \\
\hline Age $(\mathrm{yr})$ & $22.1(2.3)$ & $23.6(3.1)$ & $22.8(2.3)$ \\
Height $(\mathrm{cm})$ & $160.2(4.8)$ & $170.3(4.2)$ & $164.7(7.0)$ \\
Weight $(\mathrm{kg})$ & $50.5(3.4)$ & $63.9(9.3)$ & $56.7(9.0)$ \\
\hline
\end{tabular}

Values are presented as mean (SD). 


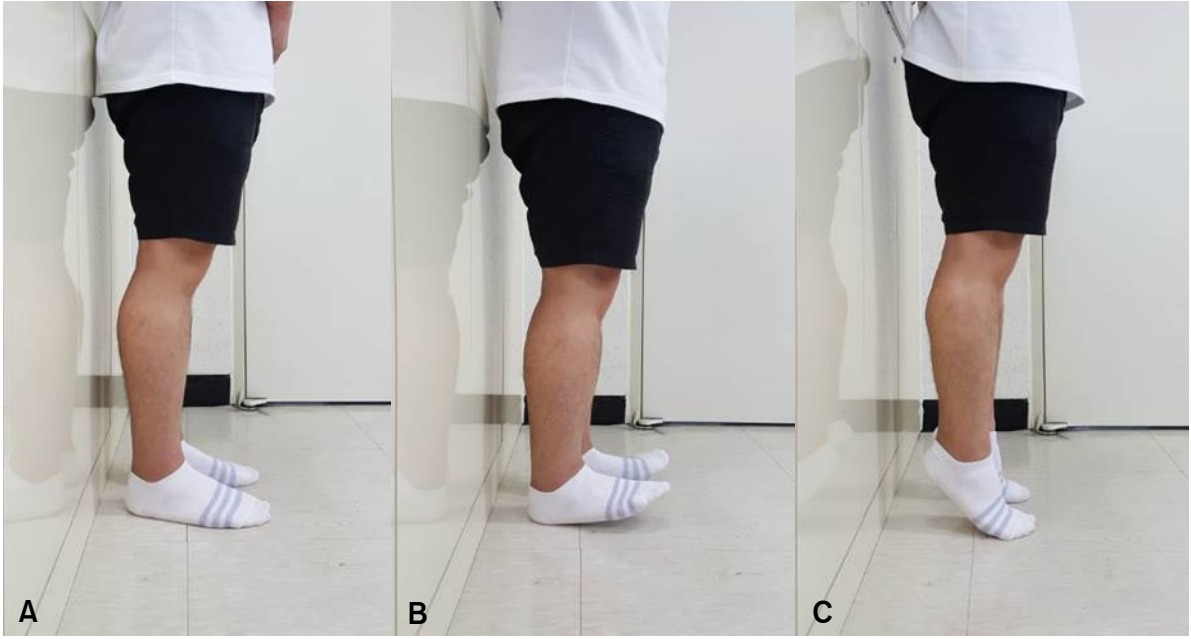

Figure 1. The fatigue duction of ankle joint muscle. (A) Start position. (B) Ankle dorsiflexion. (C) Ankle plantarflexion.

\section{Outcome measurement}

\section{Gait}

GAITRite system (CIR Systems Inc., Peakskill, NY, USA) was used to measure the gait variables. The system allowed the spatio-temporal gait parameters of subjects by having the subject walk on an electronic walkway that is 8.3 meters in length and 0.89 meters in width, and contains pressuresensitive 13,824 sensors every $1.27 \mathrm{~cm}$ connected to a personal computer. When the subject walked on the walkway, the sensors detected their footprints. The subject's gait information was collected with a sampling rate of $80 \mathrm{~Hz}$ per second. The area of the object was determined by the number of activated sensors, the distance between the sensors, and the operating/non-operating time and was transmitted to the PC via the connected cable. Information on collected temporal and spatial variables was processed with the GAITRite GOLD software ver. 3.2b (CIR Systems Inc.) [20]. The participants were measured the distance from the anterior superior iliac spine to the medial epicondyle of the ankle for inputting the subject's information in the GAITRite system (CIR Systems Inc.) before gait. Mean values on gait parameters were obtained from gait repeated three times. Gait speed of gait variables was obtained from the subject's normal walking speed in distance of 10 meters, which was repeated three times for gait analysis. Gait variables used in this study included velocity, stride length, step length, cadence, single support, and double support time.

\section{Rating of perceived exertion scale}

In the present study, the Borg scale, originally used as the fatigue criterion, was used to evaluate the degree of a head- ache, muscle pain, and chest pain. The Borg scale can easily measure fatigue without physiological measurements. Although this is a subjective measure, a person's exertion rating may provide a fairly good estimate of the level of objective physiological factors during physical activity. The scale used was as follows: 7 (very, very light), 9 (very light), 11 (moderate), 13 (moderately severe), and 15 (moderate), 15 (hard), 17 (very hard), and 19 (no more) [21].

\section{Functional reach test}

The FRT was designed by Duncan et al. [22] as a quick screen for balance problems in older adults. It is a quick and simple, single-task dynamic postural balance test. The reliability of FRT is very high with a intra-rater reliability of $\mathrm{r}=$ 0.92 and inter-rater reliability of $r=0.98$. The FRT was conducted by instructing the standing subjects to move closer to the wall (without touching it) with $90^{\circ}$ of shoulder flexion and the fist closed. The location of the 3rd metacarpal head in the starting and end positions was noted. The difference between them was recorded as the reach difference. The test was repeated 3 times.

\section{Statistical analysis}

IBM SPSS Statistics ver. 18.0 (IBM Co., Armonk, NY, USA) was used. Paired t-test was used to compare the mean of the gait parameters with or without visual information, and to compare the mean of the dynamic postural balance variables. Statistical significance was set at $\alpha=0.05$. 
Table 2. Comparison between visual block and open eyes condition on gait parameters

$(\mathrm{N}=30)$

\begin{tabular}{lcrr}
\hline \multicolumn{1}{c}{ Characteristic } & Visual block & Open eye & $\mathrm{t}(p)$ \\
\hline Step length $(\mathrm{cm})$ & $50.96(6.12)$ & $62.30(6.20)$ & $-7.49(<0.01)$ \\
Stride length $(\mathrm{cm})$ & $102.47(12.57)$ & $125.81(10.81)$ & $-8.59(<0.01)$ \\
Cadence $(\mathrm{step} / \mathrm{min})$ & $96.81(14.22)$ & $110.71(9.54)$ & $-4.65(<0.01)$ \\
Velocity $(\mathrm{cm} / \mathrm{s})$ & $83.69(19.27)$ & $115.31(17.33)$ & $-6.63(<0.01)$ \\
Single limb support (\%) & $36.96(2.70)$ & $38.20(2.80)$ & $-1.94(>0.05)$ \\
Double limb support $(\%)$ & $26.78(5.20)$ & $22.05(3.38)$ & $4.51(<0.01)$ \\
\hline
\end{tabular}

Values are presented as mean (SD).

\section{Results}

\section{Comparison of gait according to visual block after ankle fatigue}

The visual block condition produced significantly reduced step length of $11.34 \mathrm{~cm}$, the stride length of $23.34 \mathrm{~cm}$, cadence of $13.9 \mathrm{step} / \mathrm{min}$, and the velocity of $31.62 \mathrm{~cm} / \mathrm{s}$ respectively compared to the open eyes condition $(p<0.01)$, and showed significantly increased double limb support of $4.73 \%(p<0.01)$. There was no statistically significant difference between the visual block and the open eyes condition in single limb support, although the difference was decreased by $1.24 \%$ ( $p>0.05$; Table 2$)$.

\section{Comparison of dynamic balance ability with use of visual block after ankle fatigue}

In the FRT, the dynamic balance test showed a significant decrease $(4.24 \mathrm{~cm})$ in the visual block condition compared to the open eyes condition ( $p<0.01$; Table 3$)$.

\section{Discussion}

The purpose of this study was to investigate the effect of visual block on gait and balance after inducing ankle fatigue in 30 young men and women. As a result of the study, it was found that gait parameters decreased significantly in step length, stride length, cadence, and gait velocity except for single limb support, and there was an increase in double limb support. The FRT showed a significant decrease with the visual block condition.

Parijat and Lockhart [23] reported that the effects of quadriceps fatigue on gait increased the velocity of heel contact after the fatigue induction, and decreased acceleration of the body mass center in healthy adults. In addition, the range of foot dorsiflexion decreased when walking after fatigue induction, which is similar to the effect of fatigue of the ankle
Table 3. Comparison of functional reach test by visual block after ankle fatigue induction

$(\mathrm{N}=30)$

\begin{tabular}{cccc}
\hline Characteristic & Visual block & Open eye & $\mathrm{t}(p)$ \\
\hline Distance $(\mathrm{cm})$ & $32.78(6.21)$ & $37.01(6.42)$ & $-6.26(<0.01)$ \\
\hline
\end{tabular}

Values are presented as mean (SD).

joint on gait parameters in this study. The muscle fatigue showed a decrease in gait speed due to a reduction in push-off force during the stance phase of the gait cycle in young adults. These reduced gait speeds may indicate a compensatory movement for enhancing stability during walking against falling [23].

In a study on the effects of visual and auditory cognitive tasks on gait, gait in the visual deficit by cognitive tasks showed significant reduction of gait velocity, cadence, and step length compared with general gait. However, step time, swing phase, stance phase, single limb support period (\%), and double limb support period (\%) increased [24]. In the results of this study, we can see the decrease of all parameter except for single limb support and an increased double limb support when we block the visual information. This suggests that decrease in gait velocity is related to decrease of the step length and cadence [25]. In particular, Gage et al. [26] reported that the single limb support was the time that attention was required when walking. This suggests that attention concentration is increased to compensate for visual block and sensory depression during walking. In addition, the Cherng et al. [27] reported that double limb support was variable in providing control of balance during gait with motor and cognitive tasks in 48 children 4 to 6 years old. The increased double support in this study can be thought to be increased ability for the balance control during the gait with visual block.

In other studies with elderly subjects, decreased postural control by fatigue and visual-block contributed to falling in- 
cidences because the visual system plays a major role in balance control compared to proprioception or the vestibular system [28].

However, in another study, it has been reported that balance control ability is highly dependent on proprioception, however, if proprioception is compensated, the visual system plays a major role [29].

Additionally, visual block decreases postural control when participants stand on one leg with a visual block and after fatigue is induced of the ankle muscles [30]. Similar to the previous studies, our results showed balance ability through the FRT was reduced with a visual block, suggesting that visual information is used to compensate for postural control due to muscle fatigue.

The result of this study is predictable; nevertheless we hope to emphasize that weak muscle function with decreased vision in healthy adults, which is similar to the physical condition of elderly people, can be important risk factors for falls. Thus, we suggest that lower limb muscle strength and balance ability related to vision should be taken into consideration with increase age within the clinic.

The limitation of this study is that it is difficult to generalize the findings to all adults due to limitation of age, sample size, and the inclusion of healthy adults. Therefore, in future studies, it is necessary to study the change of gait in elderly people or patients with weak visual input and elderly people or patients with a history of falls. In addition, attention is increased to compensate for visual impairment. Therefore, it is necessary to study not only the effects of the visual block but also the disturbance of concentration.

The purpose of this study was to investigate the effect of visual block with ankle fatigue on gait and dynamic balance ability. In the visual block condition, the step length, stride length and cadence of gait parameters decreased, but double support time increased. Dynamic balancing ability also decreased in the visual block condition.

\section{Conflict of Interest}

The authors declared no potential conflicts of interest with respect to the authorship and/or publication of this article.

\section{References}

1. Hallemans A, Ortibus E, Meire F, Aerts P. Low vision affects dynamic stability of gait. Gait Posture 2010;32:547-51.
2. Takakusaki K. Functional neuroanatomy for posture and gait control. J Mov Disord 2017;10:1-17.

3. Paulus WM, Straube A, Brandt T. Visual stabilization of posture. Physiological stimulus characteristics and clinical aspects. Brain 1984;107:1143-63.

4. Dionyssiotis Y. Analyzing the problem of falls among older people. Int J Gen Med 2012;5:805-13.

5. Pirouzi S, Motealleh AR, Fallahzadeh F, Fallahzadeh MA. Effectiveness of treadmill training on balance control in elderly people: a randomized controlled clinical trial. Iran J Med Sci 2014;39:565-70.

6. Boyas S, Remaud A, Bisson EJ, Cadieux S, Morel B, Bilodeau M. Impairment in postural control is greater when ankle plantarflexors and dorsiflexors are fatigued simultaneously than when fatigued separately. Gait Posture 2011;34:254-9.

7. Bisson EJ, Lajoie Y, Bilodeau M. The influence of age and surface compliance on changes in postural control and attention due to ankle neuromuscular fatigue. Exp Brain Res 2014;232:837-45.

8. Roerdink M, Hlavackova P, Vuillerme N. Effects of plantar-flexor muscle fatigue on the magnitude and regularity of centerof-pressure fluctuations. Exp Brain Res 2011;212:471-6.

9. Cho HT. Analysis of gait and muscle activities on men with flatfoot in variety condition [PhD dissertation]. Seoul: Dongbang University; 2011.

10. Yoon NM, Yoon HJ, Park JS, Jeong HS, Kim G. The comparative study on age-associated gait analysis in normal Korean. J Korean Soc Phys Ther 2010;22:15-23.

11. Jung SR, Won JI. Effects of dual-task training on balance and gait performance in patients with stroke. Phys Ther Korea 2014;21: $18-27$.

12. Kong SW, Jeong YW, Kim JY. Correlation between balance and gait according to pelvic displacement in stroke patients. J Phys Ther Sci 2015;27:2171-4.

13. Granacher U, Muehlbauer T, Gollhofer A, Kressig RW, Zahner L. An intergenerational approach in the promotion of balance and strength for fall prevention-a mini-review. Gerontology 2011;57:304-15.

14. Ni Scanaill C, Garattini C, Greene BR, McGrath MJ. Technology innovation enabling falls risk assessment in a community setting. Ageing Int 2011;36:217-31.

15. Nam HS, Park DS, Kim DH, Kang HJ, Lee DH, Lee SH, et al. The relationship between muscle fatigue and balance in the elderly. Ann Rehabil Med 2013;37:389-95.

16. Soleimanifar M, Salavati M, Akhbari B, Moghadam M. The interaction between the location of lower extremity muscle fatigue and visual condition on unipedal postural stability. Eur J Appl Physiol 2012;112:3495-502.

17. Grobe S, Kakar RS, Smith ML, Mehta R, Baghurst T, Boolani A. Impact of cognitive fatigue on gait and sway among older adults: a literature review. Prev Med Rep 2017;6:88-93.

18. Heuser M, Pincivero D. The effects of stretching on knee flexor fatigue and perceived exertion. J Sports Sci 2010;28:219-26.

19. Boyas S, Hajj M, Bilodeau M. Influence of ankle plantarflexor fatigue on postural sway, lower limb articular angles, and postural strategies during unipedal quiet standing. Gait Posture 2013; 37:547-51.

20. McDonough AL, Batavia M, Chen FC, Kwon S, Ziai J. The validity and reliability of the GAITRite system's measurements: a 
preliminary evaluation. Arch Phys Med Rehabil 2001;82:419-25.

21. Chen MJ, Fan X, Moe ST. Criterion-related validity of the Borg ratings of perceived exertion scale in healthy individuals: a meta-analysis. J Sports Sci 2002;20:873-99.

22. Duncan PW, Weiner DK, Chandler J, Studenski S. Functional reach: a new clinical measure of balance. J Gerontol 1990;45: M192-7.

23. Parijat P, Lockhart TE. Effects of quadriceps fatigue on the biomechanics of gait and slip propensity. Gait Posture 2008;28: 568-73.

24. Kim JS, Ku JW. The effect of a visual and auditory cognitive task on the walking performance. Ergon Soc Korea 2008;5:133-41.

25. Beauchet O, Dubost V, Herrmann FR, Kressig RW. Stride-tostride variability while backward counting among healthy young adults. J Neuroeng Rehabil 2005;2:26.

26. Gage WH, Sleik RJ, Polych MA, McKenzie NC, Brown LA. The allocation of attention during locomotion is altered by anxiety. Exp Brain Res 2003;150:385-94.

27. Cherng RJ, Liang LY, Hwang IS, Chen JY. The effect of a concurrent task on the walking performance of preschool children. Gait Posture 2007;26:231-7.

28. Oh HJ, Youm CH, Kim TH. Effects of exercise-induced fatigue and blocked vision on postural control during upright stance. Korean J Sport Biomech 2011;21:353-9.

29. Stanmore EK, Oldham J, Skelton DA, O'Neill T, Pilling M, Campbell AJ, et al. Fall incidence and outcomes of falls in a prospective study of adults with rheumatoid arthritis. Arthritis Care Res (Hoboken) 2013;65:737-44.

30. Woo YK, Yi CH, Cho SH, Kwon HC. Effect of visual block, task type, and participation in an exercise program on static balance in the elderly. Phys Ther Korea 2003;10:1-15. 